\title{
Is care really shared? A systematic review of collaborative care (shared care) interventions for adult cancer patients with depression
}

Joanne Shaw ${ }^{1 *}$ D, Suvena Sethi ${ }^{1}$, Lisa Vaccaro ${ }^{1}$, Lisa Beatty ${ }^{2}$, Laura Kirsten ${ }^{3}$, David Kissane ${ }^{4}$, Brian Kelly ${ }^{5}$, Geoff Mitchell ${ }^{6}$, Kerry Sherman ${ }^{7}$ and Jane Turner ${ }^{8}$

\begin{abstract}
Background: Collaborative care involves active engagement of primary care and hospital physicians in shared care of patients beyond usual discharge summaries. This enhances community-based care and reduces dependence on specialists and hospitals. The model, successfully implemented in chronic care management, may have utility for treatment of depression in cancer. The aim of this systematic review was to identify components, delivery and roles and responsibilities within collaborative interventions for depression in the context of cancer.

Methods: Medline, PsycINFO, CINAHL, Embase, Cochrane Library and Central Register for Controlled Trials databases were searched to identify studies of randomised controlled trials comparing a treatment intervention that met the definition of collaborative model of depression care with usual care or other control condition. Studies of adult cancer patients with major depression or a non-bipolar depressive disorder published in English between 2005 and January 2018 were included. Cochrane checklist for risk of bias was completed (Study Prospero registration: CRD42018086515).

Results: Of 8 studies identified, none adhered to the definition of 'collaborative care'. Interventions delivered were multi-disciplinary, with care co-ordinated by nurses $(n=5)$ or social workers $(n=2)$ under the direction of psychiatrists $(n=7)$. Care was primarily delivered in cancer centres $(n=5)$. Care co-ordinators advised primary care physicians (GPs) of medication changes $(n=3)$ but few studies $(n=2)$ actively involved GPs in medication prescribing and management.

Conclusions: This review highlighted joint participation of GPs and specialist care physicians in collaborative care depression management is promoted but not achieved in cancer care. Current models reflect hospital-based multi-disciplinary models of care.
\end{abstract}

Protocol registration: The protocol for this systematic review has been registered with PROSPERO. The registration number is CRD42018086515.

Keywords: Collaborative care, Shared care, Systematic review, Depression, Cancer, Randomised controlled trial

\section{Background}

A diagnosis of cancer impacts individuals' psychological and physical wellbeing. Prevalence estimates of major depression (16\%), minor depression and dysthymia (22\%) in cancer patients are higher than in the general population. $[1,2]$ In palliative settings, the prevalence of

\footnotetext{
*Correspondence: joanne.shaw@sydney.edu.au

'The University of Sydney, Psycho-oncology Co-operative Research Group,

School of Psychology, Faculty of Science, Sydney, NSW 2006, Australia

Full list of author information is available at the end of the article
}

depression approaches 49\%. [3] Inadequate treatment of depression results in poorer adherence to anti-cancer treatments, decreased tolerance of cancer treatment side-effects, higher use of health care resources including increased hospital re-admissions, may adversely impact on interpersonal relationships and reduced overall survival. [3-9] Co-existing depression therefore poses a significant burden for patients, families and the health system. Cognitive behavioural therapy (CBT) is effective in treating depression [10] with a meta-analysis $(n=198$ studies, 22, 238 patients) reporting medium to large effect sizes

C The Author(s). 2019 Open Access This article is distributed under the terms of the Creative Commons Attribution 4.0 International License (http://creativecommons.org/licenses/by/4.0/), which permits unrestricted use, distribution, and 
sustained 6-12 months post intervention. A recent meta-analysis also confirmed the effectiveness of antidepressant medication in the treatment of depression [11] both in combination with psychological therapy and as primary treatment. [12] To guide evidence-based treatment, there has been a renewed effort to promote routine distress screening as a first step to improve detection and hence treatment for depression in cancer care. [13] Such strategies have included the development of a clinical pathway for identification and management of depression in adults with cancer, an international first. [14]

Despite increased evidence about effective treatments, many patients still do not seek treatment for their depression. For instance, a meta-analysis of 53 studies $(n=12,052)$ found in a research context less than $60 \%$ of distressed cancer patients engage in psychological treatment. [15] Uptake in routine care is even lower. [16] At a system level access to treatment is constrained by a shortfall in the psycho-oncology workforce resulting in long waiting lists and geographical disparities in access. [17-19] Practical constraints such as transport, inconvenience and cost have also been suggested. [20, 21] Normalisation of distress and attribution of somatic depression symptoms to cancer by clinicians also means depression goes untreated and patients' attitudes to mental illness are also likely to play a role. [22] Patient factors such as negative attitudes to mental health and stigma also reduce patient willingness to access treatment. [23]. To address perceived barriers, models of care that encompass systematic identification of depression in cancer patients, reduce dependence on specialist input and enable timely access to evidence-based treatment have been proposed.

The collaborative care model, based on the principles of chronic disease management, has been successfully implemented to treat medical conditions including depression. [24, 25] Core components of collaborative care for depression are: i) a multi-professional approach to patient care, ii) a structured management plan tailored to depression symptom severity, iii) scheduled patient follow-ups and iv) enhanced inter-professional communication. [26] The model endorses a multi-disciplinary approach involving joint participation of primary and specialist care physicians in planned delivery of care over and above routine discharge and referral. [23] A key aspect of effective collaborative care is case management [27] in which a member of the clinical team works closely with the main treating physician and monitors patient progress including adherence to psychological and pharmacological treatments, initiating treatment changes as necessary. [28] A meta-analysis of collaborative care interventions ( $n=37$ studies, 12,355 patients with depression receiving primary care) found that interventions that included a mental health professional as a care provider within the clinical team reported the largest effect sizes. [29]

The evidence base for collaborative care for depression among patients with cancer is rapidly developing. A recent meta-analysis of studies $(n=8)$ purporting to be collaborative care interventions concluded that the interventions were significantly more effective than usual care (standardized mean difference $=-0.49, p=0.003$ ), with remission rates for depression higher in the intervention groups at 12 months. While promising, the collaborative care interventions identified in that meta-analysis varied in content, intensity, and number of components, thus making it difficult to determine the relative contribution of each component to overall effectiveness. [30] Prior to wider implementation of the model, greater understanding of the determinants essential to model success for the treatment of depression in oncology is required. The aim of this systematic review was to determine the fidelity of the depression collaborative care models trialled in oncology to the recommended collaborative care criteria. Specifically, the review sought to identify reported intervention components, delivery models and role responsibilities.

\section{Methods \\ Study eligibility \\ Types of studies}

Studies presenting primary data from randomised controlled trials (RCTs) of adult cancer patients with major depression or a non-bipolar depressive disorder, which compared a treatment intervention designated as a collaborative (or shared) model of depression care with usual care or other control group and published in English were included. Non-randomised, single-arm, case control studies, qualitative studies and case-series reports were excluded.

\section{Participants}

Studies were eligible if participants were over 18 years of age with a current or prior diagnosis of cancer (excluding basal cell carcinoma and squamous cell carcinoma) and where the study population met a threshold for clinical depression on a validated depression measure or structured clinical interview. Studies including separate sub-group analyses of patients that met the criteria for clinical depression were also eligible for inclusion.

\section{Interventions}

Collaborative care, defined as a treatment approach integrating primary- and tertiary-level (hospital) care in the management of co-morbid depression in cancer patients.

\section{Controls}

Treatment as usual, wait-list control groups, and other treatment interventions. 


\section{Outcomes}

Studies reporting data on the efficacy of collaborative care interventions.

\section{Search strategy}

Medline, PsycINFO, CINAHL, Embase and the Cochrane Library and Central Register for Controlled Trials databases were searched using the keywords and MeSH terms [Depression/depressive disorder/ or depressive disorder, major/or dysthymic disorder/] and [Cancer or Carcinoma or Neoplasm] and [Collaborative Care or Shared Care or Integrative Care] and randomised controlled trials for English language articles published between January 2005 to January 2018 as the first collaborative care intervention for depression in cancer was published in 2005. Primary studies presenting data from RCTs of adult cancer patients with major depression or a non-bipolar depressive disorder that compared care delivered in a collaborative care model were identified. See Additional file 1: Table S1 for an example database search strategy. Reference lists of retrieved articles and previous systematic reviews were also searched for relevant publications. Searches were conducted for outcome data for published study protocols identified.

\section{Data abstraction}

Abstracts were identified and independently reviewed by two reviewers (SS and LV). Data extraction was independently conducted by three reviewers (SS, LV and JS) and coding disagreements arising were discussed and consensus coding applied. A coding framework was developed to extract the components of collaborative care based on the key criteria defining collaborative care models: i) multi-professional patient care, ii) a structured stepped care management plan, iii) scheduled patient follow-ups and iv) enhanced inter-professional communication [23] Specifically, the following information was extracted for each study: study characteristics, including study aim(s), population, design and primary outcome(s); intervention description, follow up protocols and role of health professionals. Reasons for study exclusion are listed in Additional file 2: Table S2. The review methodology undertaken adhered to PRISMA guidelines for systematic reviews [31] and the search process is summarised in Fig. 1. Risk of bias was determined based on the Cochrane criteria (Additional file 3: Table S3). [32]

\section{Results}

\section{Study characteristics}

Eight primary studies met the review inclusion criteria. Mean sample size was 281 patients (SD 161.04; range 55-500). Cancer populations included lung $(n=1)$ [33], upper gastrointestinal/liver $(n=1)$ [34], mixed breast and gynaecological $(n=2)[34,35]$ or heterogeneous $(n=4)$ [36-39]. Participants had major depressive disorder (MDD) $(n=3)$ [33, 36, 40], MDD or dysthymia $(\mathrm{n}=2)$ [37, 38], MDD, dysthymia or persistent depressive symptoms $>1$ month $(n=1)$ [35] and depressive symptoms based on self-report questionnaires only $(n=2)$ [34, 39] Greater than $60 \%$ female participation was reported in six studies $[33,35-37,39,40]$ primarily due to an over-representation of breast cancer patients. Of the included studies three were conducted by a single group in Scotland [31, 34, 35] and the remainder were conducted by separate groups in the USA. Study Characteristics are listed in Table 1.

\section{Collaborative care interventions Study setting}

In contrast to depression collaborative care models more broadly, five studies delivered the intervention within the cancer centre, [34-37, 39] with a single study conducted in primary care. [38] A further study allowed patients to choose either the cancer centre or primary care for treatment[36] and another study in advanced cancer provided an option for care to be delivered in the patient's home. [40] For two studies conducted in cancer clinics much of the care was provided via telephone/ web-based as well as face to face blended models. [34, 39] See Table 1 for study setting.

\section{Model components}

Classification of studies based on collaborative care interventions found three studies [33, 40] (collectively called the SMART studies) utilised variations of a Depression Care for People with Cancer (DCPC) intervention. [41] Two studies $[35,38]$ implemented the Improving Mood Promoting Access to Collaborative Treatment program (IMPACT), a stepped care management program developed for treatment of depression in older primary care patients and a third study (ADAPT-C [42]) adapted the IMPACT protocol to incorporate a greater patient navigation role for Latino cancer patients. [37] A further two studies reported study-specific blended face to face and telephone [39] or web-based [34] collaborative care interventions. The key components of each intervention are listed in Table 2.

All interventions provided psychoeducation and the majority $(n=7)$ included psychological therapy and/or anti-depressant medication. For the studies based on the IMPACT intervention, patient preference influenced delivery of first line psychological or anti-depressant therapy. [35, 37, 38] The SMART studies [33, 36, 40] recommended both psychological therapy and anti-depressant medication, if indicated. Similarly, treatment associated with the web-based collaborative care model was based on patient preference for cognitive behavioural therapy (CBT), antidepressant medication or both [34] and medication alone was considered first line treatment in the Kroenke trial. [39] In those studies where psychological treatment 


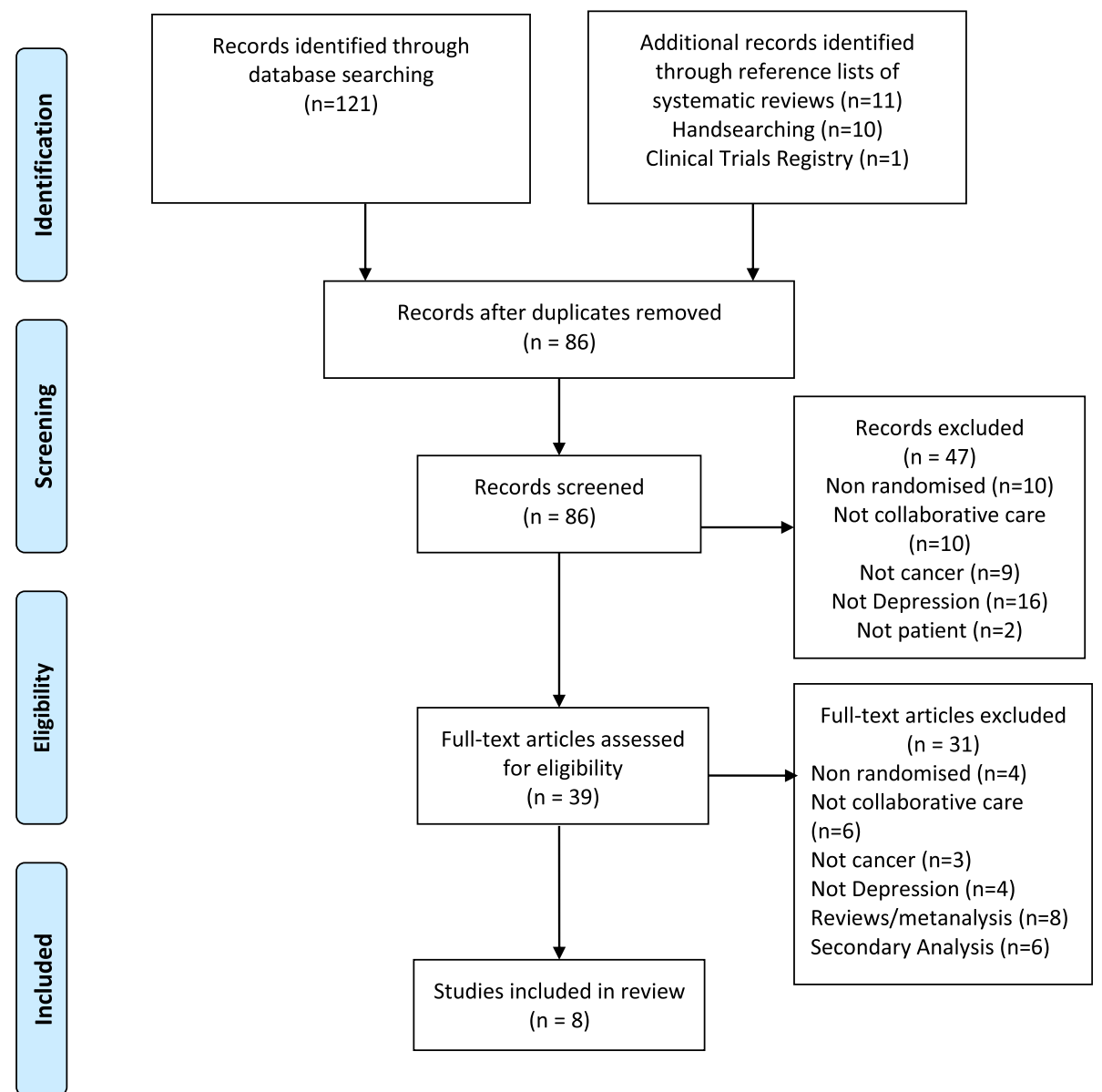

Fig. 1 (PRISMA diagram): Search process for the review (as at January 2018)

was a component of the intervention, treatment length ranged from 8 weekly sessions [33] up to 10 sessions over 3-4 months [31, 34, 35] with Problem Solving Treatment (PST) and Behavioural Activation the most common psychological interventions. [33, 35-38, 40] A single study characterised their therapy as CBT. [34] One study did not include a psychological therapy option as part of the treatment plan. [39] Six studies reported data on psychological treatments received. [33, 35, 36, 39, 40] Rate of uptake for psychological treatment in the intervention groups were higher than control groups and ranged from 5 to 98\%. [33, 35-37, 40] Studies reliant on patient preferences for either psychological or pharmacological depression treatment reported lower rates of uptake (5-43\%). $[35,37]$ Similarly, rates of antidepressant use were higher than controls and ranged from 35 to $85 \%$ of participants at 6 months. [33, 36, 37, 39, 40]

Five studies included training in intervention delivery for care co-ordinators [33, 34, 36, 37, 40] and a single study documented training for oncologists responsible for antidepressant management. [35] Care co-ordinators' fidelity to the intervention manual was formally assessed in four studies [33, 36, 37, 40] although fidelity assessment and ongoing education were not included as part of the protocol for studies where the role of prescribing was delegated to oncologists and GPs.

Inter-professional roles within the collaborative care model

With respect to multi-professional involvement, all studies included a care co-ordinator and a mental health specialist as the primary members of the care team. Across studies, delivery of the psychological/psychoeducation components of the intervention as well as patient liaison and assessment of treatment adherence was undertaken by an appropriately trained nurse $(n=4),[33,36,39,40]$ social worker $(n=2)[35,37]$ or a psychologist $(n=1)$ [34] with one study including both a nurse and a psychologist. [38] The care co-ordinators all underwent formal study-specific training and had weekly [33, 34, 36-40] or bi-weekly [35] supervision to review patient progress and initiate treatment adjustments, as required. Supervision was generally provided by a psychiatrist $(n=7)$, although in one study a clinical psychologist was the appointed supervisor. [32] 


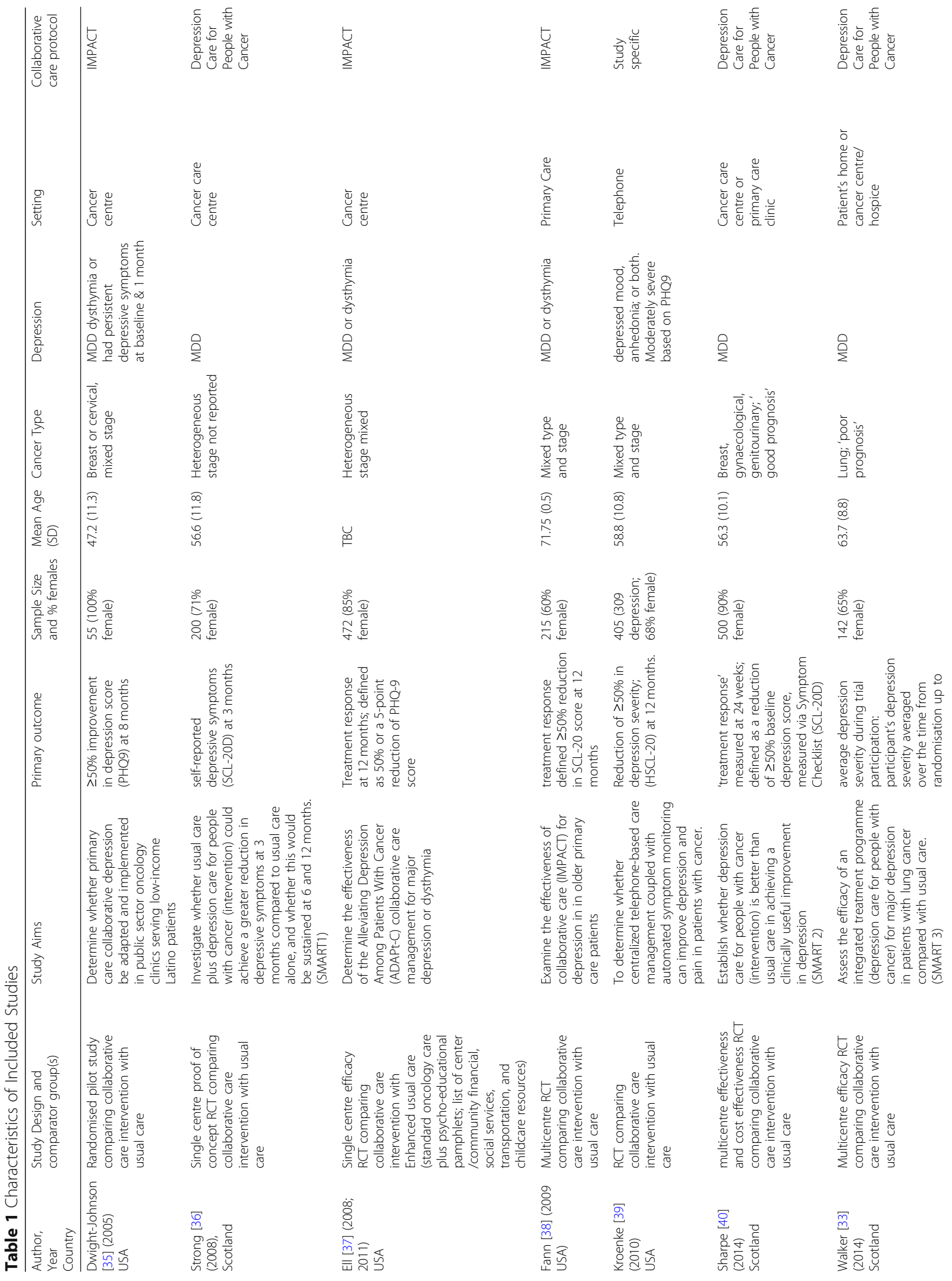




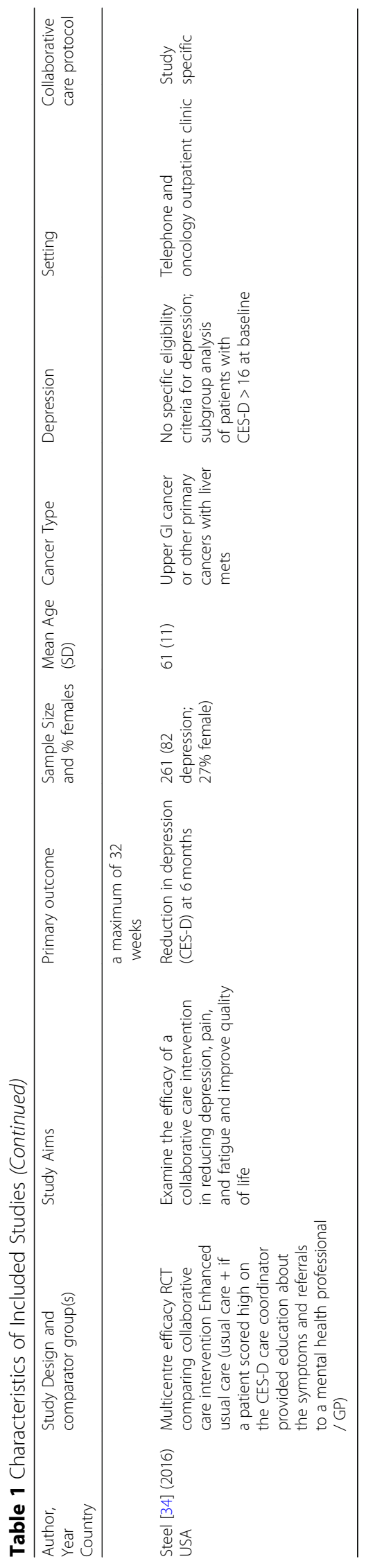




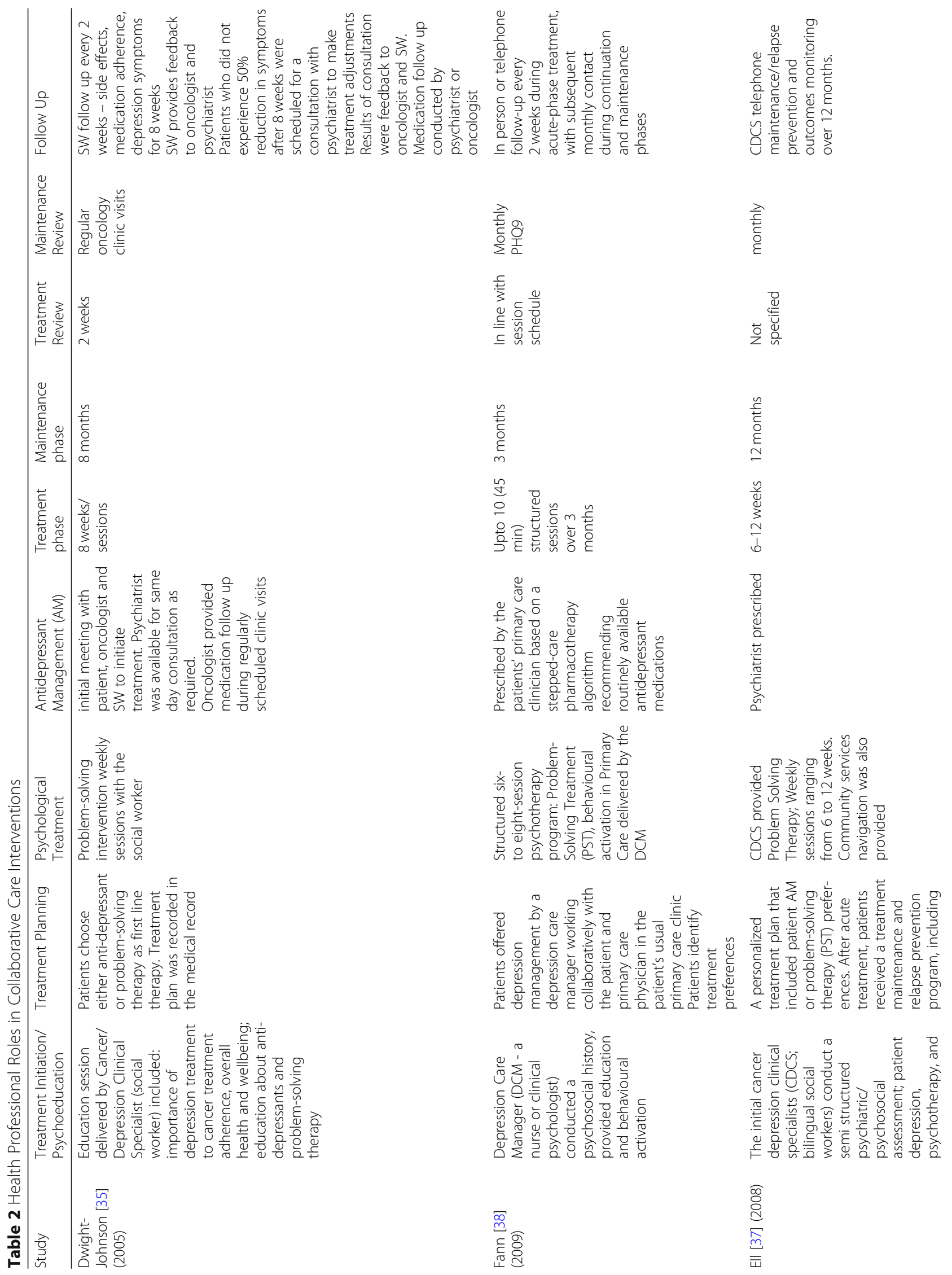




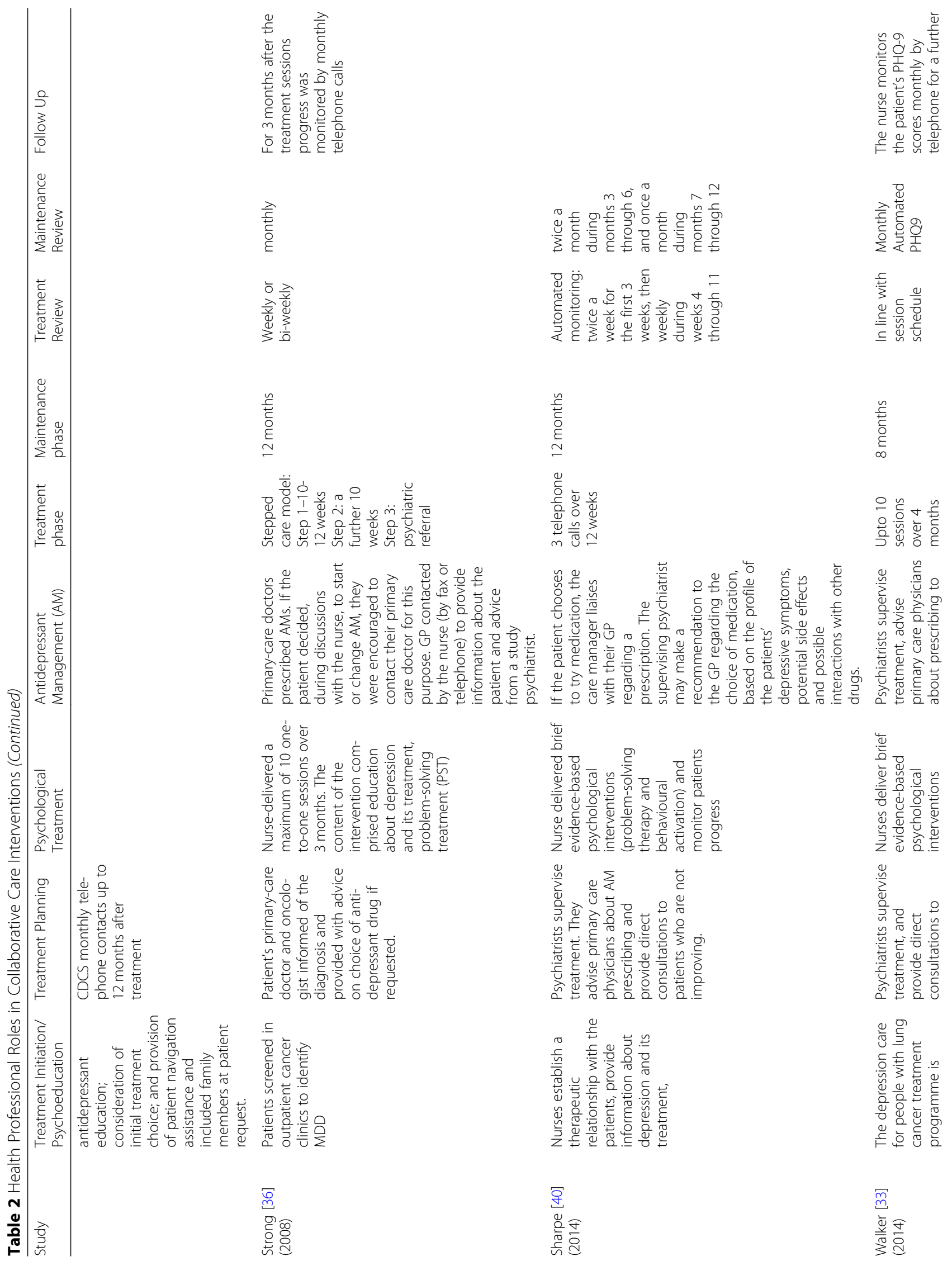




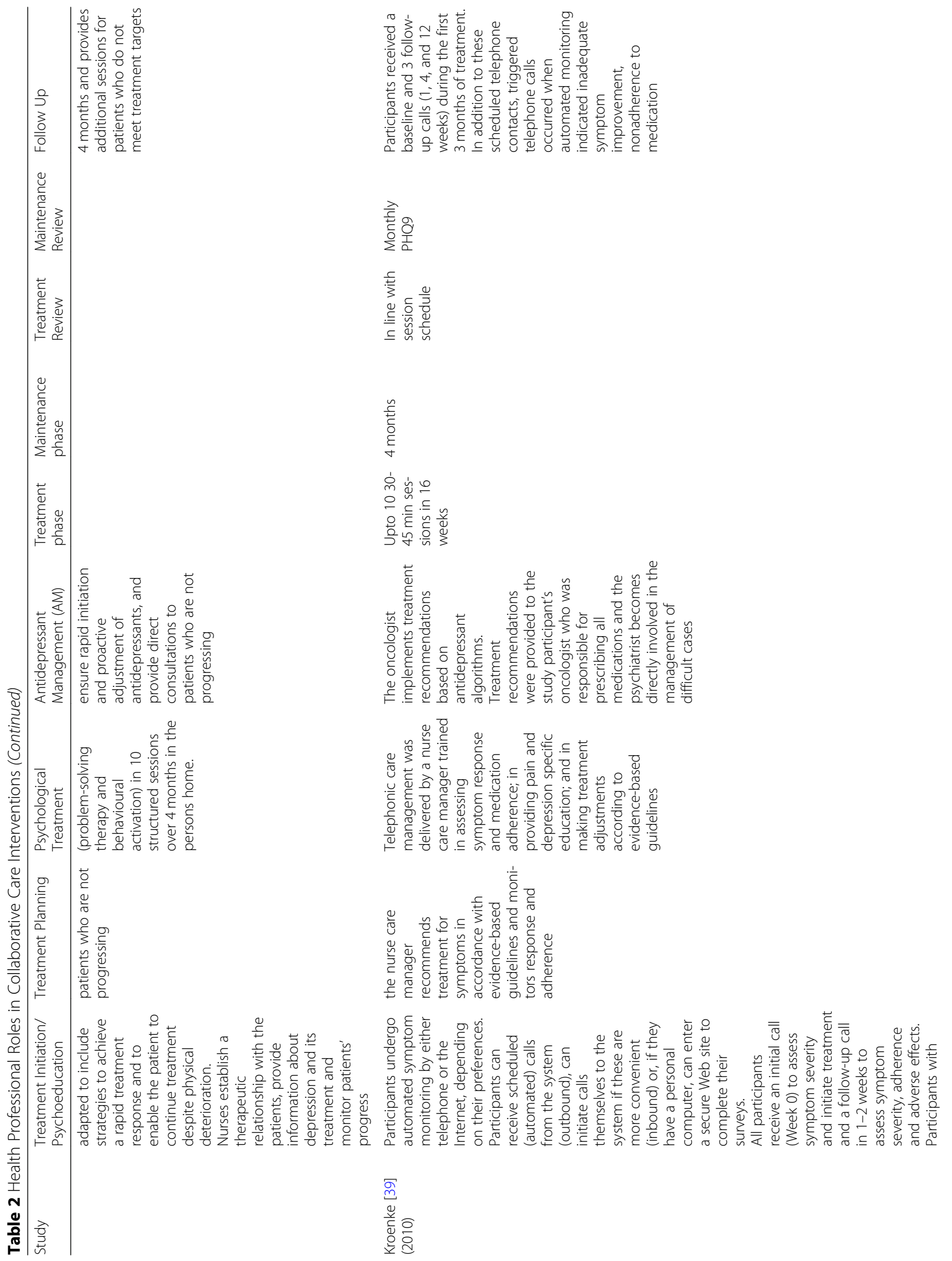




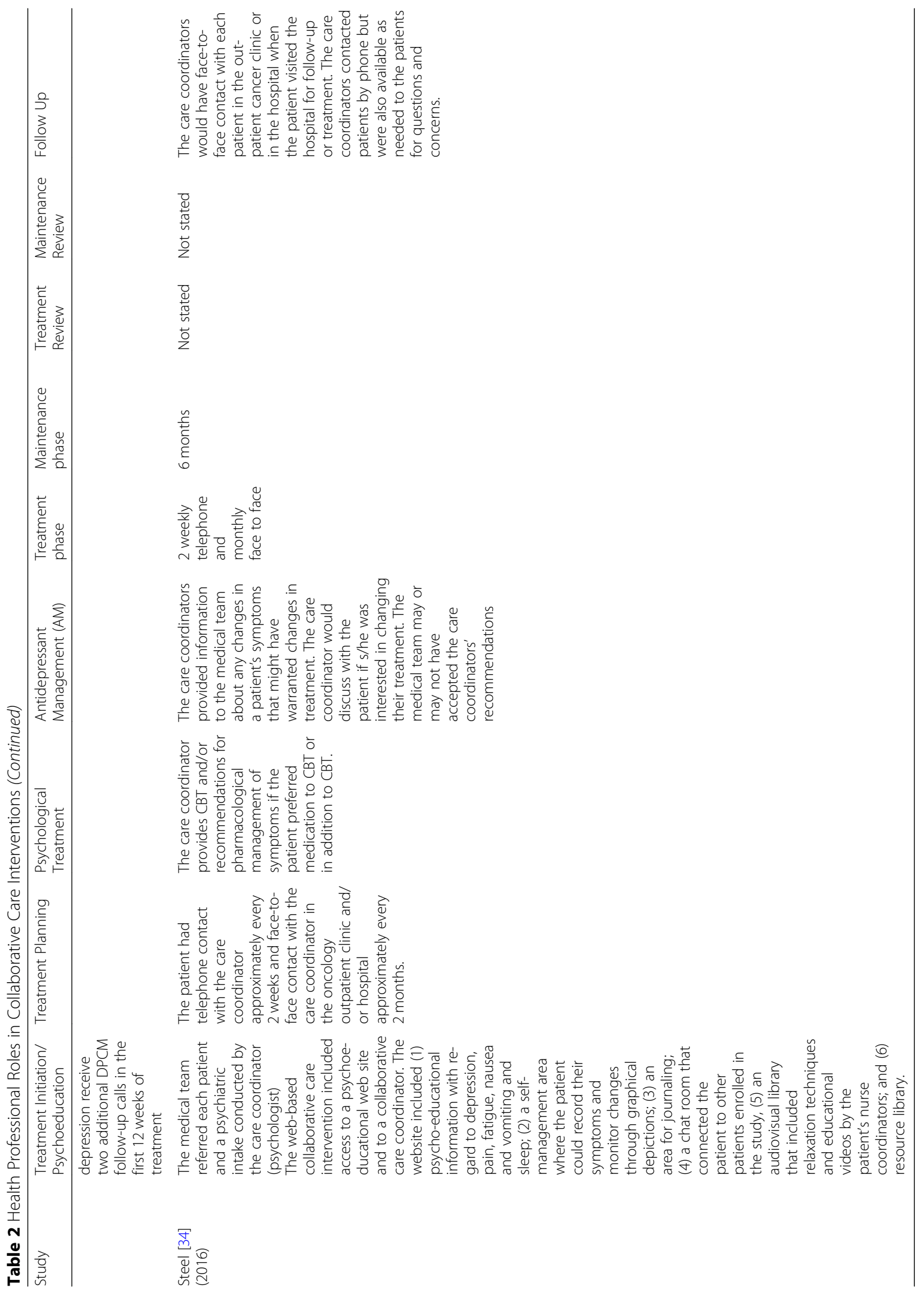


Despite being classified as collaborative care interventions, studies deviated from the model in terms of wider inter-professional involvement in treatment, with four studies reporting oncologists were typically informed of the patient's diagnosis but had minimal engagement with respect to treatment decision-making. [33, 36, 37, 40] In one study [35] the psychiatrist provided advice to the oncologist with respect to anti-depressant treatment adjustment/ follow up and in a second study, the oncologist followed evidence-based anti-depressant prescribing and management algorithms, with little input from the psychiatrist. [39] Similarly, three studies reported little or no primary care (GP) involvement in treatment decision-making and no documented contact with the GP by the treating team. [35, 37, 39] In the studies utilising the DPCP intervention (SMART studies), [31, 34, 35] although the GPs were responsible for anti-depressant prescribing, clinical decisions and recommendations for medication treatment and/or adjustment were made by the psychiatrist, with little engagement with the GP around treatment planning. A single study reported GP prescribing based on evidence-based algorithms [38] and one study divided prescribing responsibility between the medical team and the patient's GP, although the role of each clinician was not clearly defined. [34] Table 3 lists the health professional roles across studies.

\section{A structured management plan tailored to depression symptom severity}

Consistent with the collaborative care model, all interventions reported implementation of a structured management plan. The treatment phase ranged from 6 to 16 weeks and included weekly [33, 34, 36-40] or bi-weekly [35] review meetings between the care co-ordinator and psychiatrist to monitor patient progress and make treatment adjustments as required. Seven studies incorporated a stepped care protocol [36, 40] and/or documented criteria for dose escalation and/or psychiatry consultation. [31, 33-35, 37] Of the five studies reporting follow-up post the initial treatment phase, management varied from 3 to 4 months $[33,36]$ to $8-12$ months $[37,38,40]$ with non-responders being offered additional psychological treatment booster sessions. [33, 36, 37, 40] Table 2 outlines intervention management plans.

\section{Scheduled patient follow-ups}

While the majority of interventions included weekly or fortnightly face to face follow up during the treatment phase, one study relied on 3 telephone calls over 12 weeks to supplement automated symptom monitoring [39] and a second study included 2 weekly telephone calls and monthly face to face follow up. [34] The maintenance phase ranged from 3 to 12 months with monthly follow up, [31, 34-37, 40] although one study linked follow up to regular (unspecified) oncology clinic visits. [37] The web-based symptom monitoring study did not include any maintenance or relapse prevention strategies after the initial treatment period, although symptoms continued to be monitored online. [34] Follow up protocols are listed in Table 2.

\section{Enhanced inter-professional communication}

Across all studies communication was facilitated by the care co-ordinator and there were clear communication protocols between the care co-ordinator and the supervisory psychiatrist/psychologist regarding treatment review and follow up. However, there was limited inter-professional collaboration to establish the treatment plan and discuss progress incorporated into any of the collaborative care intervention models. For the three SMART studies, where the GP was responsible for prescribing anti-depressants, the care co-ordinator sent detailed written reports outlining depression scores, progress and any treatment recommendations to each GP. Although these studies did not detail any formal communication with oncologists, copies of reports were forwarded to other relevant professionals. [33, 36, 40]. Similarly, for studies where the oncologist was responsible for anti-depressant prescribing, the protocols included feedback from the care co-ordinator to the oncologist, [35, 39] although the nature of this feedback was only documented in one study. [37] For two studies the inter-professional communication plan was not specified. [34, 38] Across studies, engagement with the psychiatrist was typically limited to their review of the reports, with recommendations subsequently communicated by the care co-ordinator, although in one study the psychiatrist was available to provide same day treatment decision support on an ad-hoc basis. [35] Case conferencing with the non-mental health care providers was not a feature in any of the collaborative care models described. Communication protocols are listed in Table 3.

\section{Quality of study reporting}

Risk of bias was assessed based on Cochrane criteria. (Additional file 3: Table S3). Randomisation processes were well described in six studies. [33, 35-38, 40]. Across studies patients and the health professionals delivering care were not blinded to group allocation, however researchers assessing outcomes remained blinded. Sample size considerations were reported for four studies [33, 36, $39,40]$ and met by three studies. [36, 39, 40] Rates of attrition at the end of the initial treatment phase (3-4 months) were reported for three studies [33, 35, 36] and ranged from 2 to 20\%. Attrition during the maintenance phase (6-9 months) was reported for five studies [33, 34, 37-39] and ranged from 4 to $57 \%$. Long term attrition rates $\geq 12$ months was reported for 4 studies (9-45\%) [37-40]. 


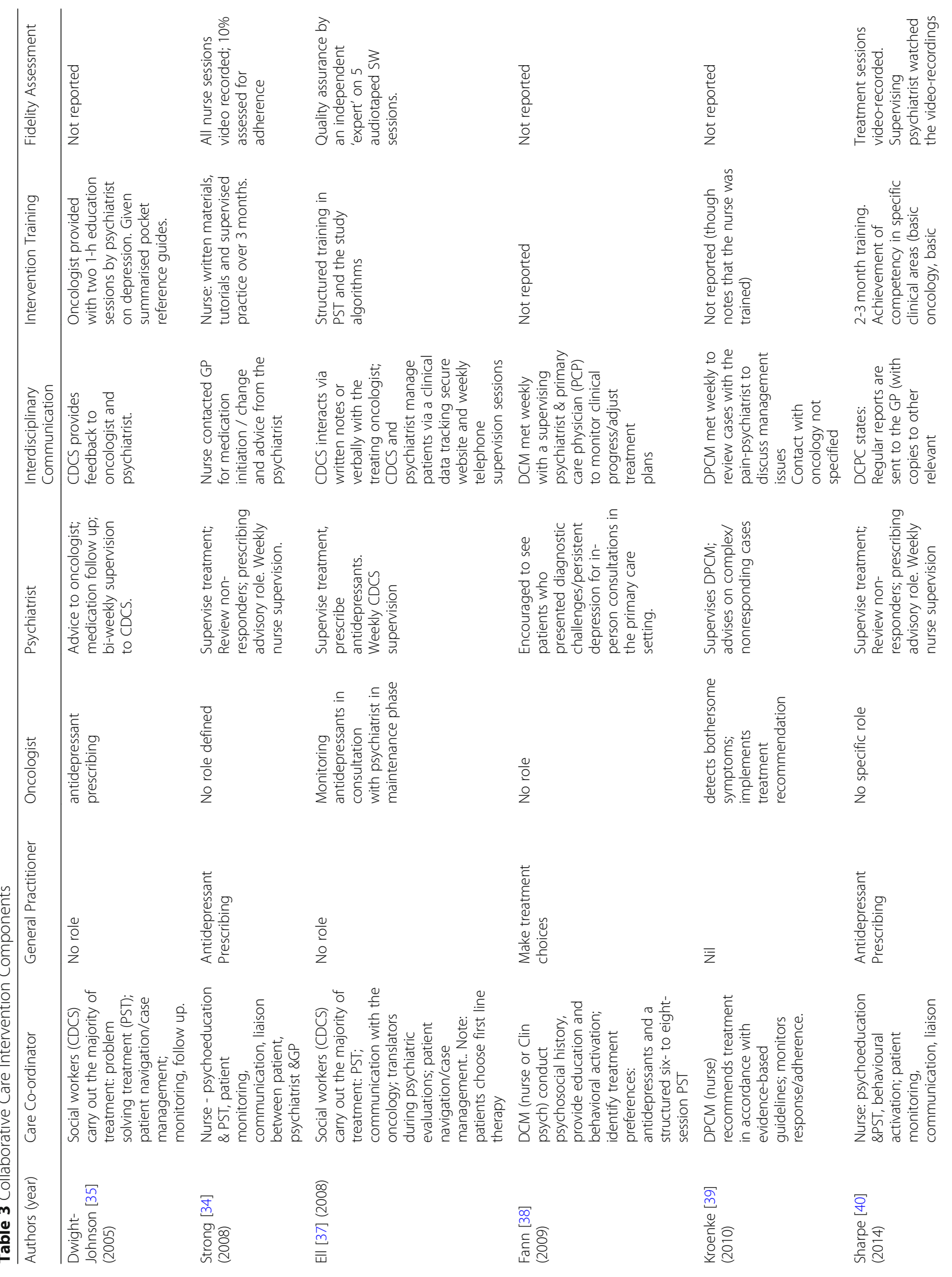




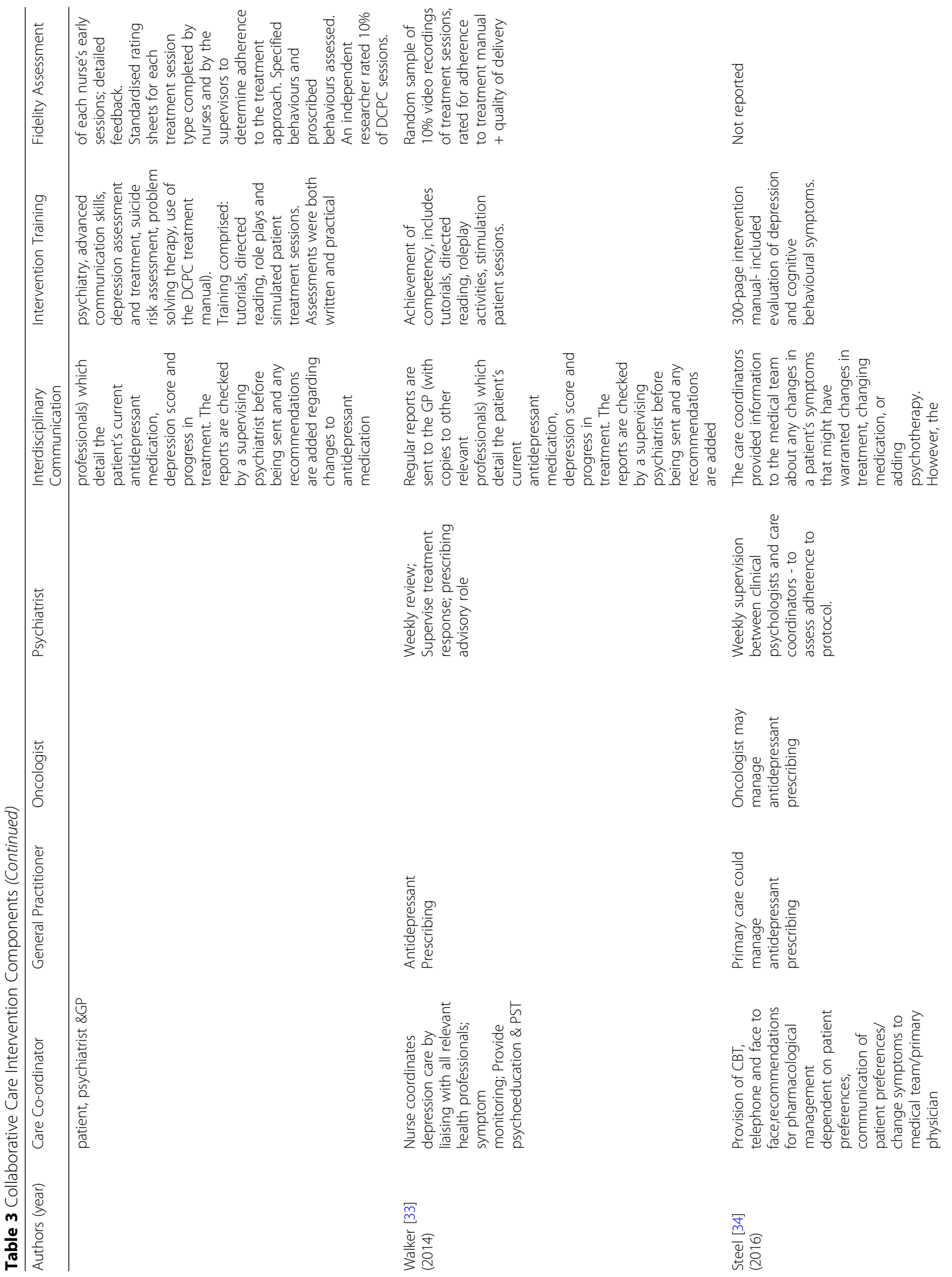




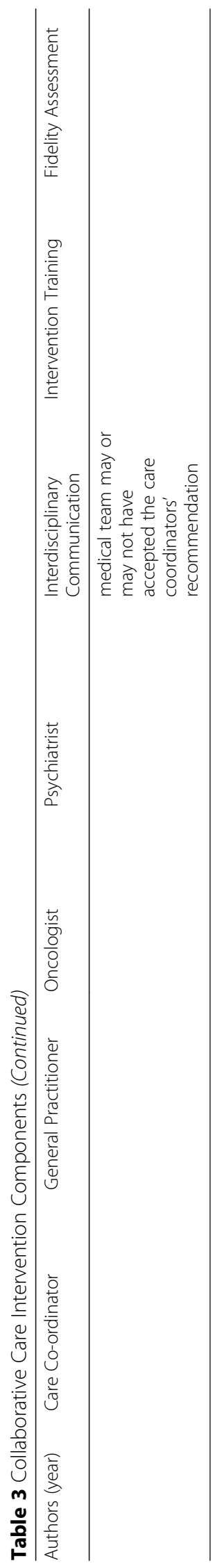


Analysis was based on intention to treat for seven studies. [33-36, 38-40]

\section{Discussion}

Collaborative care interventions have the potential to improve management of depression for people with cancer. However, before wider implementation, there are several important questions that need to be addressed, including clearer definition of the elements of collaborative care (including roles, communication processes, governance), which collaborative care components are essential to treatment outcomes and who is best placed to deliver care. We sought to explore these questions by systematically describing the components of depression collaborative care interventions. Eight collaborative care RCTs were identified in this review. Five interventions were developed specifically for patients with cancer and three interventions were adapted from a more general collaborative care intervention for management of depression in older patients.

All studies identified in our review highlighted the benefit of systematic, intensive depression treatment and ongoing symptom monitoring, and reported decreased depression symptoms in comparison to usual care. However, in contrast to collaborative care interventions more generally, the review found the interventions were primarily delivered in the hospital setting rather than in primary care. While the hospital-based care model makes sense for those receiving ongoing cancer treatment, this model does little to address the preference of patients for care closer to their home and issues of access, particularly for those patients not in active treatment. [43] Similarly, the continued dependence on hospital specialists for the management of patients does not ameliorate the scarcity of psycho-oncology resources available in many cancer services.

In the literature, collaborative care is used to describe a range of interventions of varying intensity from simple interventions to encourage compliance with medication (typically delivered via telephone) to complex multi-component interventions that incorporate psychological and pharmacological treatments and intensive monitoring and follow up. [29] The studies identified in this review were consistent with this heterogenous depiction of collaborative care, ranging from symptom monitoring and patient navigation, anti-depressant adherence models to interventions involving proactive follow-up by care co-ordinators working closely with psychiatrists integrating pharmacological medical and psychological treatments as part of individualized treatment plans. As a result, treatment dose within the interventions identified varied in level of intensity as well as whether patients were able to access psychological and pharmacological treatment. Importantly, a number of collaborative care models were reliant on anti-depressant medication as the primary treatment. This is of concern given current gold standard depression treatment recognises the importance of combined cognitive behavioural therapy and anti-depressant medication, [44] and the fact that patients may be reluctant to take antidepressant medication. [45] Despite this, all studies documented clear pathways to patient management and follow up protocols to ensure patients had access to appropriate levels of care.

Despite the focus of hospital-based care, the single study in our review that provided care in the primary care setting, [38] demonstrated the acceptability among people diagnosed with cancer to attend primary care appointments for depression management. Other studies such as the Sharpe study [40] purported to provide the option for patients to choose depression care in either the cancer centre or primary care, however in truth this was limited to anti-depressant prescribing rather than overall psychological treatment and care coordination, and the authors did not evaluate differences in outcome based on whether GPs or oncologists were responsible for anti-depressant management. Lack of GP engagement across studies is surprising given the increasing evidence GPs can play an important role in collaborative care models in terms of continuity of care and reduced patient burden due to excessive travel. From a health service perspective, inclusion of GPs will in part address some of the psycho-oncology workforce shortages that mean that many patients do not receive depression treatment in a timely manner. Under this model of care, less complex patients' pharmacological treatment can be managed in the community, with psychiatry treating only those patients with persistent symptoms.

A key feature of collaborative care models is a team-based approach to delivery of care. Previous reviews have highlighted the importance of a multi-disciplinary approach to care, with inclusion of case managers with mental health training reported to increase the efficacy of the model. [29] Our review found in the context of cancer, care co-ordination was primarily undertaken by nurses trained to deliver the intervention. However, given their limited specialist mental health training and consistent with the findings of previous work conducted by our Group that nurses are inadequately equipped to deliver depression care, [46] there was a high level of psychiatry case management incorporated into all studies, with psychiatrists providing both staff supervision and treatment decision-making. The time commitment required for ongoing psychiatry oversight limits long term sustainability of the proposed model in routine care as it is predicated on sufficient psychiatry staff to provide the high level of supervision. Modifications of the model to include other (non-medical) mental health staff such as clinical psychologists, such as demonstrated in the study by Steel [34] in the delivery of psychological treatment and supervision of 
care co-ordinators in a stepped care model, with psychiatric review reserved for those patients in need of more intensive psychiatric care, is one way to reduce is one way to reduce the need for routine psychiatry oversight.

Additionally, over-reliance on specialist psychiatrist involvement for treatment decision-making further underscores the perception among oncology clinicians that the mental wellbeing of patients is not the responsibility of the cancer team and is further reinforced when oncologists are informed of their patient's depression diagnosis but not engaged in any delivery of depression care. Similarly, although primary care physicians provided pharmacological treatment to their patients as part of the intervention, we were unable to identify any studies that incorporated specific training for GPs about prescribing and follow up. Upskilling of non-mental health providers in evidence-based management of depression is essential for a truly collaborative model to be sustainable. However, in the studies identified in this review, communication pathways between hospital-based care providers and GPs failed to engage GPs in shared decision-making as much of the communication was via written reports or telephone calls initiated by the care co-ordinator (nurse). This resulted in passive engagement with treatment decisionmaking whereby GPs implemented recommendations for individual patients rather than establishing ongoing communication where treatment decisions were jointly shared between care providers, taking into account not only the patient's cancer and current depression episode, but each patient's wider medical and social history available from their primary care provider. Attention to clinical governance and management of shared care is necessary to achieving positive patient outcomes. The funding models and organisation of primary care and specialist care vary internationally and influence the governance of such models. This context necessitates closer examination of the processes and ingredients of findings regarding collaborative care when evaluating transferability to diverse settings. Evidence from "shared care" for primary mental disorders indicates that substantial cross-organisational commitment is required, with consistently delivered interventions, and with close attention to staff selection, training and supervision. [47] Implementation and longterm sustainability of shared care models is contingent on funding models that support clinical pathways that incorporate primary and tertiary care, the introduction of information technology to facilitate information sharing, and collaborative interdisciplinary practice models.

\section{Study limitations}

The results of this review need to be considered in light of a number of limitations. Firstly, we were unable to conduct the planned meta-analysis to compare between intervention components due to the small number of studies identified. The review however does extend the recent meta-analysis conducted by $\mathrm{Li}$ and colleagues [30] that demonstrated overall efficacy of the model, as we were able to qualitatively explore the components of each intervention and compare the roles of health professionals across interventions. Our analysis of intervention components was however limited by the intervention descriptions detailed in study publications; the authors may have used a more detailed intervention protocol when conducting their study and explicit references to previously published standardised protocols were included in assessment of interventions. Similarly, we were unable to determine the quality of training provided as part of the interventions or assess the quality of care provided by each member of the care team. Generalisability of review results are also limited to interventions published in English. All studies identified also excluded patients with co-morbid psychiatric and substance use disorders, limiting generalisability of the study results given the high prevalence of co-occurring mental health conditions in the general population. Despite these limitations, this review highlights that, collaborative care interventions for depression in oncology do vary according to key criteria such as level of inter-professional engagement and communication, inclusion of psychological and pharmacological treatment options and documented follow up.

\section{Conclusions}

In the context of research, collaborative care interventions for the management of depression in cancer have demonstrated efficacy over usual care. However, the sustainability of the level of hospital staff engagement raises questions about the model's utility in routine care. Current models of care reflect hospital-based multi-disciplinary models of care. Greater engagement with oncology and primary care is required to reduce the over-reliance on specialist psychiatry services. Models that utilise existing community-based services, including clinical psychologists in partnership with GPs have the potential to enhance depression care. Given the aim of collaborative models is to provide more care in the community, future models need to consider the training needs of primary care providers and there needs to be greater emphasis placed on inter-professional communication and shared decision-making.

Future research is required to explore patient and provider acceptability as well as pragmatic non-inferiority trial designs that incorporate process evaluations to identify implementation strategies and practice change required to move these models of care from research into clinical practice. 


\section{Additional files}

Additional file 1: Table S1. example search strategy PsycINFO search. (DOCX $13 \mathrm{~kb}$ )

Additional file 2: Table S2. Reasons for study exclusion from the review at both title and abstract and fulltext screening. (DOCX $17 \mathrm{~kb}$ )

Additional file 3: Table S3. Assessment of Bias and Outcomes. (DOCX $16 \mathrm{~kb})$

\section{Abbreviations}

ADAPT-C: Alleviating Depression Among Patients with Cancer intervention; CBT: Cognitive behavioural therapy; DCPC: Depression Care for People with Cancer intervention; GPs: General practitioners/primary care physicians; IMPACT: Improving Mood - Promoting Access to Collaborative Treatment intervention; MDD: Major depressive disorder; PST: Problem solving Treatmen

\section{Acknowledgements}

Not applicable.

\section{Funding}

No funding was obtained for this study. The Psycho-oncology Co-operative Research Group is supported by Cancer Australia through their Support for Cancer Clinical Trials Funding Scheme

\section{Availability of data and materials}

Search strategy and excluded studies are included in the additional files. A detailed list of Collaborative Care Intervention Components and an Assessment of Study Bias are also included as additional files. All other data is included in the manuscript and associated tables and figures.

\section{Authors' contributions}

JS, LB, LK, DK, BK, GM, KS, and JT contributed to the overall study rationale, design and methods development. JS, SS and LV conducted the review. All authors contributed to the drafting of the manuscript and approved the final version

\section{Ethics approval and consent to participate}

Not applicable.

\section{Consent for publication}

Not applicable.

\section{Competing interests}

The authors declare that they have no competing interests.

\section{Publisher's Note}

Springer Nature remains neutral with regard to jurisdictional claims in published maps and institutional affiliations.

\footnotetext{
Author details

${ }^{1}$ The University of Sydney, Psycho-oncology Co-operative Research Group, School of Psychology, Faculty of Science, Sydney, NSW 2006, Australia. ${ }^{2}$ College of Medicine \& Public Health, Flinders Centre for Innovation in Cancer, Adelaide, SA, Australia. ${ }^{3}$ Nepean Cancer Care Centre, Sydney, NSW, Australia. ${ }^{4}$ Department of Psychiatry, Monash University, Melbourne, Vic, Australia. ${ }^{5}$ School of Medicine \& Public Health, The University of Newcastle (UoN), Callaghan, NSW, Australia. ${ }^{6}$ Faculty of Medicine, The University of Queensland, Herston, Qld, Australia. ${ }^{7}$ Centre for Emotional Health, Department of Psychology, Macquarie University, Sydney, NSW, Australia. ${ }^{8}$ Discipline of Psychiatry, School of Medicine, The University of Queensland, Herston, Qld, Australia.
}

Received: 17 September 2018 Accepted: 4 February 2019 Published online: 14 February 2019

\section{References}

1. Mitchell AJ, Chan M, Bhatti $\mathrm{H}$, et al. Prevalence of depression, anxiety, and adjustment disorder in oncological, haematological, and palliative-care settings: a meta-analysis of 94 interview-based studies. Lancet Oncol. 2011 12:160-74.

2. Mehnert A, Brähler E, Faller $\mathrm{H}$, et al. Four-week prevalence of menta disorders in patients with Cancer across major tumor entities. J Clin Oncol. 2014;32:3540-6.

3. Walker J, Sawhney A, Hansen $\mathrm{CH}$, et al. Treatment of depression in people with lung cancer: a systematic review. Lung Cancer. 2013;79:46-53.

4. Pratt LA, Druss BG, Manderscheid RW, Walker ER. Excess mortality due to depression and anxiety in the United States: results from a nationally representative survey. Gen Hosp Psychiat. 2016;39:39-45.

5. Brown LF, Kroenke K, Theobald DE, Wu J, Tu W. The Association of Depression and Anxiety with health-related quality of life in Cancer patients with depression and/or pain. Psycho-Oncology. 2010;19:734-41.

6. Hanrahan NP, Bressi S, Marcus SC, Solomon P. Examining the impact of comorbid serious mental illness on rehospitalization among medical and surgical inpatients. Gen Hosp Psychiat. 2016;42:36-40.

7. Prieto JM, Blanch J, Atala J, Carreras E, Rovira M, Cirera E, et al. Psychiatric morbidity and impact on hospital length of stay among hematologic Cancer patients receiving stem-cell transplantation. J Clin Oncol. 2002;20:1907-17

8. Pinquart M, Duberstein PR. Depression and cancer mortality: a metaanalysis. Psychol Med. 2010;40:1797-810.

9. Dorros SM, Card NA, Segrin C, Badger TA. Interdependence in women with breast Cancer and their partners: an inter-individual model of distress. J Consult Clin Psychol. 2010;78:121-5.

10. Faller $H$, Schuler M, Richard $M$, et al. Effects of psycho-oncologic interventions on emotional distress and quality of life in adult patients with Cancer: systematic review and meta-analysis. J Clin Oncol. 2013;31:782-93.

11. Cipriani A, Furukawa TA, Salanti G, et al. Comparative efficacy and acceptability of 21 antidepressant drugs for the acute treatment of adults with major depressive disorder: a systematic review and network metaanalysis. Lancet. 2018:391:1357-66.

12. Li M, Kennedy EB, Byrne N, et al. Management of Depression in patients with Cancer: a clinical practice guideline. J Oncol Pract. 2016:12:747-56.

13. Bultz B, Travado L, Jacobsen PB, et al. President's plenary international psycho-oncology society: moving toward cancer care for the whole patient. Psycho-oncology. 2015. 2014;24:1587-93.

14. Butow PN, Price MA, Shaw J, et al. Clinical pathway for the screening, assessment and management of anxiety and depression in adult cancer patients: Australian guidelines. Psycho-Oncology. 2015;24:987-1001.

15. Brebach $R$, Sharpe $L$, Costa $D$, et al. Psychological intervention targeting distress for cancer patients: a meta-analytic study investigating uptake and adherence. Psycho-Oncology. 2016;25:882-90.

16. Faller $\mathrm{H}$, Weis J, Koch $\mathrm{U}$, et al. Utilization of professional psychological care in a large German sample of cancer patients. Psycho-Oncology. 2017;26: 537-43.

17. Eakin EG, Strycker LA. Awareness and barriers to use of cancer support and information resources by HMO patients with breast, prostate, or colon cancer: patient and provider perspectives. Psycho-Oncology. 2001 10:103-13.

18. Absolom K, Holch P, Pini S, et al. The detection and management of emotional distress in cancer patients: the views of health-care professionals. Psycho-Oncology. 2011:20:601-8.

19. Dilworth S, Higgins I, Parker V, et al. Patient and health professional's perceived barriers to the delivery of psychosocial care to adults with cancer: a systematic review. Psycho-Oncology. 2014;23:601-12.

20. Carlson LE, Angen M, Cullum J, et al. High levels of untreated distress and fatigue in cancer patients. Brit J Cancer. 2004;90:2297-304.

21. Corboy D, McDonald J, McLaren S. Barriers to accessing psychosocial support services among men with cancer living in rural Australia: perceptions of men and health professionals. International J Men's Health. 2011:10:163

22. Kissane DW. Unrecognised and untreated depression in cancer care. Lancet Psychiat. 2014;1:320-1

23. Okuyama T, Endo C, Seto T, et al. Cancer patients' reluctance to disclose their emotional distress to their physicians: a study of Japanese patients with lung cancer. Psycho-Oncology. 2008;17:460-5.

24. Hickman M, Drummond N, Grimshaw J. A taxonomy of shared care for chronic disease. J Public Health. 1994;16:447-54.

25. Archer J, Bower P, Gilbody S, et al. Collaborative care for depression and anxiety problems. Cochrane Database Syst Rev. 2012;10:CD006525. 
26. Gunn J, Diggens J, Hegarty K, Blashki G. A systematic review of complex system interventions designed to increase recovery from depression in primary care. BMC Health Serv Res. 2006;6:88.

27. Gilbody S, Whitty P, Grimshaw J, Thomas R. Educational and organizational interventions to improve the management of depression in primary care: a systematic review. JAMA. 2003;289:3145-51.

28. Von Korff M, Goldberg D. Improving outcomes in depression : the whole process of care needs to be enhanced. BMJ. 2001;323:948-9.

29. Gillbody S, Bower P, Fletcher J, Richards D, Sutton AJ. Collaborative care for depression: a cumulative meta-analysis and review of longer-term outcomes. Arch Intern Med. 2006;166:2314-21.

30. Li M, Kennedy EB, Byrne N, et al. Systematic review and meta-analysis of collaborative care interventions for depression in patients with cancer. Psycho-Oncology. 2017;26:573-87.

31. Moher D, Liberati A, Tetzlaff J, Altman DG. The Prisma group. Preferred reporting items for systematic reviews and meta-analyses: the PRISMA statement. PLoS Med. 2009;6:e1000097.

32. Higgins JPT, Altman DG, Gøtzsche PC, et al. The Cochrane Collaboration's tool for assessing risk of bias in randomised trials. BMJ 2011;343:d5928

33. Walker J, Hansen CH, Martin P, et al. Integrated collaborative care for major depression comorbid with a poor prognosis cancer (SMaRT Oncology-3): a multicentre randomised controlled trial in patients with lung cancer. Lancet Oncol. 2014;15:1168-76.

34. Steel Jl, Geller DA, Kim KH, et al. Web-based collaborative care intervention to manage cancer-related symptoms in the palliative care setting. Cancer. 2016:122:1270-82

35. Dwight-Johnson M, Ell K, Lee PJ. Can collaborative care address the needs of low-income Latinas with comorbid depression and cancer? Results from a randomized pilot study. Psychosomatics. 2005;46:224-32.

36. Strong V, Waters R, Hibberd C, Murray G, Wall L, Walker J, et al. Management of depression for people with cancer (SMaRT oncology 1): a randomised trial. Lancet. 2008;372(9632):40-8.

37. Ell K, Xie B, Quon B, et al. Randomized controlled trial of collaborative care management of depression among low-income patients with cancer. J Clin Oncol. 2008:26:4488-96.

38. Fann JR, Fan MY, Unutzer J. Improving primary care for older adults with cancer and depression. J Gen InternMed. 2009;24:S417-S24.

39. Kroenke K, Theobald D, Wu J, et al. Effect of telecare management on pain and depression in patients with cancer: a randomized trial. JAMA. 2010;304:163-71.

40. Sharpe M, Walker J, Hansen $\mathrm{CH}$, Martin P, et al. Integrated collaborative care for comorbid major depression in patients with cancer (SMaRT Oncology-2): a multicentre randomised controlled effectiveness trial. Lancet. 2014;384: 1099-108.

41. Walker J, Sharpe M. Depression Care for People with Cancer: a collaborative care intervention. Gen Hosp Psychiat. 2009:31:436-41.

42. Ell K, Quon B, Quinn DI, Dwight-Johnson M, Wells A, Lee PJ, et al. Improving treatment of depression among low-income patients with cancer: the design of the ADAPt-C study. Gen Hosp Psychiat. 2007;29:223-31.

43. Shepherd $\mathrm{L}$, Goldstein D, Whitford $\mathrm{H}$, et al. The utility of videoconferencing to provide innovative delivery of psychological treatment for rural Cancer patients: results of a pilot study. J Pain Symptom Manag. 2006;32:453-61.

44. Malhi GS, Bassett D, Boyce P, et al. Royal Australian and new Zealand College of Psychiatrists clinical practice guidelines for mood disorders. ANZ J Psychiat. 2015:49:1087-206.

45. Wu SM, Brothers BM, Farrar W, Andersen BL. Individual counseling is the preferred treatment for depression in breast Cancer survivors. J Psychosoc Oncol. 2014;32:637-46

46. Turner J, Kelly B, Clarke D, et al. A randomised trial of a psychosocial intervention for cancer patients integrated into routine care: the PROMPT study (promoting optimal outcomes in mood through tailored psychosocial therapies). BMC Cancer. 2011;11:48.

47. Kelly BJ, Perkins DA, Fuller JD, Parker SM. Shared care in mental illness: a rapid review to inform implementation. Int J Ment Health Syst. 2011;5:31.

\section{Ready to submit your research? Choose BMC and benefit from:}

- fast, convenient online submission

- thorough peer review by experienced researchers in your field

- rapid publication on acceptance

- support for research data, including large and complex data types

- gold Open Access which fosters wider collaboration and increased citations

- maximum visibility for your research: over $100 \mathrm{M}$ website views per year

At BMC, research is always in progress.

Learn more biomedcentral.com/submissions 